\title{
Frontières
}

\section{Nouvelles parutions - Études sur la mort}

Novembre 2004

\section{Ngoc-Thanh Phan Nguyen}

Volume 17, numéro 1, automne 2004

URI : https://id.erudit.org/iderudit/1073621ar

DOI : https://doi.org/10.7202/1073621ar

Aller au sommaire du numéro

Éditeur(s)

Université du Québec à Montréal

ISSN

1180-3479 (imprimé)

1916-0976 (numérique)

Découvrir la revue

Citer ce document

Phan Nguyen, N.-T. (2004). Nouvelles parutions - Études sur la mort :

novembre 2004. Frontières, 17(1), 99-100. https://doi.org/10.7202/1073621ar d'utilisation que vous pouvez consulter en ligne.

https://apropos.erudit.org/fr/usagers/politique-dutilisation/ 
NOUVELLES PARUTIONS ÉTUDES SUR LA MORT

(Novembre 2004)

Ngoc-Thanh Phan Nguyen

AÏSSEL, Selim (2004), Le livre occidental de la vie et de la mort, Paris, Oxus.

ALBOM, Mitch (2004), La dernière leçon: comment un vieil homme face à la mort m'a appris le goût de vivre. Traduit de l'américain, Paris, R. Laffont, coll. «Aider la vie».

ANDRIAT, Frank (2004), Depuis ta mort, Paris, Hachette.

BANVILLET, François (dir.), (2004), Prier au temps du deuil, Prières, Paris, Chalet, coll. «Fenêtre sur la prière».

BELMIN, Joël et AMALBERTI, Francine (2004), L'infirmière et les soins aux personnes âgées, Issy-les-Moulineaux (Hauts-de-Seine), Masson, coll. "Savoir et pratique infirmière».

BOLES, J.-M. et LEMAIRE, F. (dir.) (2004), Fin de vie en réanimation, Paris, Elsevier.

BOULOUQUE, Clémence, (2004), Mort d'un silence, Paris, Gallimard, coll. «Folio».

CAROL, Anne (2004), Les médecins et la mort: $X I X^{e}-X X$ siècle, Paris, Aubier, coll. "Historique».

CHAGNON, François et MISHARA, Brian L. (dir.) (2004), Évaluation de programmes en prévention du suicide, Sainte-Foy, Presses de I'Université du Québec.

CHARLES, Monique (2004), Dieu es-tu là ?: au cœur de la vie, de la mort, de la douleur, Paris, Lettres du monde.

CLAVANDIER, Gaëlle (2004), La mort collective: pour une sociologie des catastrophes, Paris, CNRS Éditions, coll. «CNRS-Sociologie».

CONSEIL DE L'EUROPE (2004), Peine de mort: après l'abolition, Strasbourg, Éditions du Conseil de l'Europe.

DESMET, Marc (2004), Souffrance et dignité humaine, Namur (Belgique), Fidélité.

DUPRAT, François (2004), Mon cousin dans la mort, Darnetal (Seine-Maritime), Éditions Petit à petit.

DUVAL, Armand (2004), Dieu est plus fort que la mort: célébrations pour des obsèques. Nouv. éd. rev. et augm., Paris, Médiaspaul.

FLÈCHE, Christian et LAGARDET, Jean-Jacques (2004), L'instant de la guérison, Barret-le-Bas (Hautes-Alpes), Souffle d'or, coll. «Champ d'idées».

FLIS-TRÈVES, Muriel (2004), Deuil de maternité, Paris, Calmann-Lévy.

GALLAY, Isabelle et PERCIN, Laurence de (2004), Face au décès, comment faire?: prévoir son départ: testament, contrat obsèques; gérer l'après-mort: funérailles, formalités administratives, succession; faire son deuil: étapes de la souffrance et du vide, Paris, Vuibert, coll. "Guid'utile».

GEFFRÉ, Claude, JAVARY, Cyril et LABIDI-MAÏZA, Mehrézia (2004), Y a-t-il quelque chose après la mort? Introduction, Paris, Éd. De I'Atelier, coll. «Questions de vie».

GUEULETTE, Jean-Marie (2004), Reste auprès de moi, mon frère: vivre la mort d'un ami, Paris, Cerf, coll. «Épiphanie». 
HAUSSAIRE-NIQUET, Chantal (2004), Le deuil périnatal: le vivre et l'accompagner, Barret-sur-Méouge, (France), Le Souffle d'or.

HIRSCH, Emmanuel (dir.) (2004), Face aux fins de vie et à la mort: éthique et pratiques professionnelles au cœur du débat, Paris, Vuibert, coll. «Espace éthique».

HOFER, Marcel (2004), Explique-moi la mort...: guide pour accompagner l'enfant en famille et en catéchèse, Bruxelles, Lumen vitæ, coll. «Pédagogie catéchétique».

HAECHLER, Jean (2004), Naître, vivre et passer: la mort démythifiée, Paris, L'Harmattan.

HOUZIAUX, Alain (dir.) (2004), Doiton légaliser l'euthanasie?, Paris, Éd. de l'Atelier, coll. "Questions de vie".

ISCHER, François (2004), L'homme face à la mort, Paris, La Martinière.

JACQUEMIN, Dominique (2004), Éthique des soins palliatifs, Paris, Dunod, coll. "Action sociale».

KÜBLER-ROSS, Elisabeth et KESSLER, David (2004), Leçons de vie: deux experts de la mort et des phases terminales nous révèlent les mystères de la vie. Traduit de l'américain, Paris, Pocket, coll. «Pocket. Best».

LAMBIN, Michèle (2004), Aider à prévenir le suicide chez les jeunes: un livre pour les parents, Montréal, Éd. de l'Hôpital Sainte-Justine, coll. "Hôpital Sainte-Justine pour les parents».
LENOIR, Frédéric et TONNAC, JeanPhilippe de (dir.) (2004), La mort et l'immortalité: encyclopédie des savoirs et des croyances sur la mort et l'immortalité, Paris, Bayard.

LEGROS, Bérengère (2004), L'euthanasie et le droit: état des lieux sur un sujet médiatisé, Bordeaux, Études hospitalières, coll. «Essentiel».

LÉONARD, André-Mutien (2004), La mort et son au-delà, Paris, Presses de la Renaissance.

LEVERRIER, Michel (2004), L'impossible de l'accès à la parole: quatre histoires cliniques: autisme, mutisme psychotique, dépression infantile et deuil chez l'enfant, RamonvilleSaint-Agne (Haute-Garonne), Érès, coll. «Psychanalyse et clinique».

LETHIERRY, Hugues (dir.) et al., (2004), Parler de la mort... et de la vie: un tabou dans l'éducation?, Paris, Nathan, coll. «Repères pédagogiques".

MARGUERAT, Daniel et MÜLLER, Denis (dir.) (2004), Mourir... et après?: questions de vie, Genève (Suisse), Labor et Fides.

MARIN, Isabelle (2004), Allez donc mourir ailleurs!: un médecin, l'hôpital et la mort, Paris, Buchet Chastel.

MELLINI, Laura, GODENZI, Alberto et PUY, Jacqueline de (2004), Le sida ne se dit pas: analyse des formes de secret autour du VIH-sida, Paris, L'Harmattan, coll. "Le travail du social».
MISHARA, Brian L. et TOUSIGNANT, Michel (2004), Comprendre le suicide, Montréal, Presses de l'Université de Montréal.

MORGAN, Edmund, MORGAN, Marie et al. (2004), Une passion américaine: prison et peine de mort. Traduit de l'américain, Paris, les Empêcheurs de penser en rond, coll. "Vu d'Amérique».

DUPOUEY, Patrick (dir.) (2004), La mort: introduction, choix de textes, commentaires, vade-mecum et bibliographie, Paris, Flammarion, coll. «GF. Corpus».

PERELBERG, Rosine Jozef (dir.) (2004), Violence et suicide. Traduit de l'anglais, Paris, Presses universitaires de France.

PLON, Michel et REY-FLAUD, Henri (dir.) (2004), La pulsion de mort: entre psychanalyse et philosophie, Ramonville-Saint-Agne (HauteGaronne), Erès.

POHIER, Jacques (2004), La mort opportune: les droits des vivants sur la fin de leur vie, Paris, Seuil, coll. «Points. Essais».

RICHARD, Marie-Sylvie (2003), Soigner la relation en fin de vie: familles, malades, soignants, Paris, Dunod.

RAIMBAULT, Ginette (2004), Parlons du deuil, Paris, Payot, coll. «Essais».

ROGAK, Lisa (2004), Death Warmed over: Funeral Food, Rituals, and Customs from around the World, Berkeley, Ten Speed Press.

RUSZNIEWSKI, Martine (2004), Face à la maladie grave: patients, familles, soignants, Paris, Dunod, coll. "Action sociale».
SHAPIRO, Debbie (2004), Vos douleurs et vos maladies ont un sens: la guérison corps esprit, Paris, G. Trédaniel.

VANDEKERCKHOVE, Lieven (2004), La punition mise à nu: pénalisation et criminalisation du suicide dans l'Europe médiévale et d'Ancien Régime. Traduit du néerlandais, Louvain-la-Neuve (Belgique), Academia-Bruylant.

VELUT, Stéphane (2004), L'illusoire perfection du soin: essai sur un système, Paris, L'Harmattan, coll. "Questions contemporaines".

VERSPIEREN, Patrick et RICHARD, Marie-Sylvie (2004), À propos de I'euthanasie, Paris, Desclée De Brouwer.

WOLFELT, Alan Dean (2004), Understanding Your Grief: Ten Essential Touchstones for Finding Hope and Healing Your Heart, Laguna hills (CA), Companion Press.

WOODWARD, John (2004), Teen Suicide, San Diego, Greenhaven Press.

WUNNENBERG, Kathe (2004), Pleurer l'enfant que je n'ai jamais connu: traverser l'épreuve d'une fausse couche ou d'un deuil périnatal avec l'aide de Dieu. Traduit de I'américain, Lautenbach (HautRhin), Famille je t'aime.

YAHYA, Harun (2004), La mort, la résurrection et l'enfer, Paris, Lora.

YUFIT, Robert I. et LESTER, David (2004), Assessment, Treatment, and Prevention of Suicidal Behavior, New York, Wiley. 